\title{
Patient Consent in Dentistry: Are we Legally Safe?
}

\author{
Anil Sharma', Anuj Chhabra ${ }^{2}$, Arun Sharma ${ }^{3}$, C Bopiah ${ }^{4}$
}

\begin{abstract}
As with other walks of life the profession of dentistry saw unprecedented change during the last century. Amongst other things, litigation, which is a part of everyday life, has evolved and spread its growing influence on dentistry. Though the litigation and claims are prevalent in almost all countries however, the western countries have been at the forefront of Dental negligence claims. In a way, it is a sign of an informed and mature society.

Dentistry in India has been relatively immune to the effects of litigation against dental professionals for a multitude of reasons. But its will not be long before it envelops every aspect of dentistry in India. Dental legislation has its pros and cons; it serves to be a system, which protects patients as well as professionals, but as with other spheres of law, will be subjected to abuse. This article attempts to address the core issue of patient consent, which is where the majority of dental negligence claims arise.
\end{abstract}

Keywords: Consent, Presumed consent, Ethics, Consent form

\section{${ }^{1}$ Professor}

Dept of Prosthodontics

ITS Dental College, Hospital \& Research Centre

Greater Noida, U.P.

2Senior Lecturer

Dept of Prosthodontics

ITS Dental College, Hospital \& Research Centre

Greater Noida, U.P.

\section{${ }^{3}$ Professor}

Dept of Pedodontics \& Preventive Dentistry

ITS Centre for Dental studies \& Research

Murad Nagar, U.P.

${ }^{4}$ Honorary Lecturer

Faculty of Dental Surgery

The Royal College of Surgeons of England

United Kingdom

\section{Contact Author}

Dr. Anuj Chhabra

i_ac80@yahoo.co.in

J Oral Health Comm Dent 2011;5(2)68-72

\section{INTRODUCTION}

]

aw has been an integral part of Human Civilisation since time immemorial. It is a fact of life that has quietly and steadily grown along with the evolution of man. Today, we live in a world of ever-increasing consumerism and its product has been a maturing legal system. It serves to keep checks and balances on trades and industries and the people who serve them.

Law in the sphere of dentistry is an established concept in developed countries but remains in its infancy in India. As India steadily sheds its tag of being a developing nation and promises to be a world leader in the years to come, it is a matter of time before we fall prey rightfully or wrongfully to an ever-evolving legal framework for the health care industry including dentistry. We, as professionals in society do have an ethical and moral obligation to protect our consumers, society as a whole. Dental professionals have been relatively immune to the effects of dental-negligence with convictions for wrongdoings being far and few in India.

Is it time to lay down the blueprint for Dento-legal frameworks? Are we opening a can of worms or are we protecting our profession and more importantly our precious patients? Dental law is in itself worth volumes of text but a starting point would be establishing the concept of Patient Consent.

\section{WHAT IS CONSENT?}

The Webster's Dictionary defines consent as "to give assent or approval" (1). The over simplification of Webster's definition however does not and should not apply to the field of Dentistry. The British Dental Association "Ethics in Dentistry" advice sheet is a case in point (2). The concept of medical informed consent is evident already in the Hippocratic Oath, which clearly illustrates the notion that respect is an integral part of the relationship between patients and health care professionals in the pledge, "First, do no harm" (3).

\section{HOW WOULD YOU DEFINE CONSENT TO DENTISTRY?}

Informed consent is not a signature on a consent form. It is not a single event, it is a process of dialogue between the dentist and the patient. Continuing throughout the course of treatment. The process of dialogue additionally should be 
documented and be a combination of objectivity and subjectivity.

The British Dental Association (BDA) 'ethics in dentistry' advice sheet defines the process of Expressing consent as "A patient gives express consent when he or she indicates orally or in writing consent to undergo examination or treatment or for personal information to be processed" (2).

\section{WHAT ARE THE TYPES OF CONSENT?}

The BDA Ethics in Dentistry (2) further lays down the following definitions.

- Implied consent: Where the patient indicates agreement to examination by lying in the dental chair and opening the mouth.

- Informed consent: Requires a full explanation of the nature, purpose and material risks of the proposed procedures in a language that the patient understands. The patient should have the opportunity to consider the information and ask questions in order to arrive at a balanced judgement of whether to proceed with the proposed treatment.

- Valid Consent: For consent to be valid it must be specific, informed and normally be given by a patient or a parent or a guardian.

- Written consent: Most countries in which dental law is an evolved concept require written consent to be taken for major procedures, examples being in Implantology, wisdom teeth removals, sedation etc. Written consent is all the above with a signature of the patient essential.

Written consent is important but cannot be considered a substitute for obtaining valid consent (4). This brings us to the obvious question of the right age of patient for giving consent.

\section{IS THERE AN AGE FOR CONSENT?}

As a general rule, consent for treatment of minors, the intellectually disabled or others not considered legally competent to make decisions on their own behalf, should be obtained from a parent, guardian or attorney (as appropriate) who must be provided with the same amount of information as would be required for adult consent. Mature minors may validly consent to treatment. In Australia, there is statutory provision for the consent of a minor over the age of 14 years. In other jurisdictions the common law position is that consent to treatment may be given by a minor provided that there is understanding of its nature. Nevertheless, it is prudent to seek parental consent whenever it is possible to obtain it, to avoid misunderstanding and conflict, notwithstanding the legal position.

In the United Kingdom the legal age of consent is 16 . However if in the dentist's opinion a child under the age of 16 who are of sufficient maturity and intelligence to understand fully the nature of treatment proposed and its ramifications are also entitled to consent to treatment.Substitute decision-making processes are also needed for adults incapable of providing consent. The multitude of disputes and court cases over the past few decades have brought into focus the principles and fundamentals of consent. For very often consent is the Achilles tendon of disputed treatment rather than treatment itself. Further, consent in its elaborate form is evidence of appropriate standards of health care being followed by the health care worker.

\section{OBTAINING CONSENT}

The key to obtaining and being able to establish informed consent is evidence of the nature and scope of the discussions or discussion held by a dentist with his or her patient before a proposed procedure or treatment. Among the benefits of engaging patients actively in an informed consent dialogue is the establishment of an atmosphere of trust, thereby strengthening the dentist-patient relationship and reducing the likelihood of miscommunication and litigation (5). A case in point is the relatively intricate and technically demanding field of Implantology, the infancy of its science brings along with it low levels of awareness amongst the general population. This reinforces the need for a continued comprehensive dialogue with the patient under treatment or parents or guardians as the case may be. Informed consent is a legal and practical reality, and communication is the cornerstone of that process (6).

King J. in his study found that the form in use for General dental services in the UK requiring the patient's signature is mistakenly regarded as a consent form by two-thirds of dental practitioners, an example of misunderstood implied consent (7). A patient gives consent he/ she allows themselves to be examined (implied consent). It is only the beginning of a process, which through comprehensive and documented dialogue evolves into informed consent. Additionally, King J. found that most of the information given to the patient was verbal, the majority of patients did not receive written treatment plans and in some cases the decisions on treatment plans were made by the dentists with limited or no participation of the patient. In the court of law these could amount to negligent practice.

Interestingly in his study King J quoted a patient saying, "routine treatment seems to have less consent and the more complex stuff consent is properly sought". Consent should not be assumed to have been obtained by the dentist for he or she regards the procedure as routine. Seeking consent is better described as joint decisionmaking': the patient and heath professional need to come to an agreement on the best way forward, based on the patient's values and preferences and the health professionals clinical knowledge.

\section{KEY FACTORS}

The Health Care Consent Act, 1996

Ontario (8) included obtaining the following salient features for informed consent:

- Nature of proposed treatment

- Expected benefits

- Material risks and side effects...

- Alternative courses of action

- Consequences of not having the proposed treatment

- Answers to any questions the patient has regarding the proposed treatment

- Cost of the treatment 
Information should be provided in layperson's terms to the patient. For example, many patients may not understand the dental term gingivectomy, but they would understand if someone would have told them they were having their diseased gum tissue surgically removed.

\section{WHICH RISKS SHOULD BE DISCUSSED?}

Dentists cannot anticipate every possible risk, nor should they be required to discuss every conceivable risk. Statutes and case law often use the phrase material risks when describing which risks should be reviewed prior to treatment. Material risks are defined as those risks that are most relevant to the patient (i.e., the most common and the most serious). For example, if an oral surgeon knows that a certain percentage of patients will experience an infection after a procedure, it would be appropriate to discuss that risk with the patient. Similarly, if a patient could experience permanent nerve damage as a result of an inferior alveolar nerve block, the risk should be discussed with the patient, even though the percentage of patients experiencing permanent paresthesia is minimal. Informed consent is necessary when there is a significant chance of problems or where potential problems are devastating (9). Dentists should also explain there may be other potential risks, but that the most common and most serious risks are being reviewed with the patient. Poor communication about risks can lead to patients making potentially dangerous decisions about their medical treatment(10).

When discussing alternative treatments with patients, dentists are not obligated to discuss every possible alternative. Informed consent statutes often use the term "reasonable" alternatives. A dentist should also explain why alternative treatments are not recommended.

In concluding the discussion, dentists should encourage patients to ask questions and then document that the patient was given the opportunity to ask questions.
Additionally, the dentist may wish to suggest a referral for a second opinion if the patient appears undecided or if prudence would dictate a second opinion in light of the seriousness of the treatment.

\section{HOW SHOULD PERTINENT INFORMATION BE SUPPLIED?}

Information should be provided through discussion followed by written information.

\section{VERBAL DISCUSSION}

The treating dentist should conduct a faceto-face discussion with the patient. This provides the dentist with an additional opportunity to interact with the patient and to strengthen the dentist-patient relationship. Unfortunately, no matter how well the dentist documents the patient education process, much of the discussion component does not get documented in the chart. This is why discussions should be supplemented with written information.

\section{WRITTEN INFORMATION}

Dental practices often use patient information sheets or educational brochures to help describe various proposed treatments. When selecting or composing such educational materials, dentists should ensure that the information outlined on page one of this newsletter is included (diagnosis, proposed treatment, anticipated benefits, risks, etc.).

Written educational materials are valuable primarily because they provide excellent documentation of what was communicated to the patient about the proposed treatment. Therefore, dentists or their staff should be sure to document the provision of these materials. Moreover, there is good evidence that many clinicians are themselves poor communicators (11). Patients have consistently protested about the failure to communicate effectively and this is believed to be one of the key causes of increasing patient litigation and even more time consuming formal complaints. Written materials are also valuable in that they allow the dentist to communicate with other family members who may not be present during informed consent discussions. Lastly, written materials are helpful because they reiterate previously discussed information, which may enhance patient comprehension and remain documented evidence for future reference.

\section{LANGUAGE BARRIERS}

Dentists should consider potential language barriers when communicating with patients regarding proposed treatments. Dentists may wish to develop written educational materials in different languages to meet the needs of their patient population. Additionally, they may wish to hire multilingual staff or explore sources for interpreters to assist in patient education discussions.

\section{CONSENT}

Oftentimes, much emphasis is placed on obtaining patients' written consent and less attention is devoted to the educational process. Such emphasis is misplaced. While written evidence of consent is helpful in defending dental malpractice claims, the informed consent process itself, is far more important than the form. That being said, dentists should evaluate those situations where a consent form may be necessary. Consent forms should conclude with a statement that the patient has had an opportunity to have all of his/her questions answered, and that the patient understands he/she may seek a second opinion.

\section{Special considerations and Precautions}

- Verbal or written consent. Complex treatments ideally should have written consent from the patient or legal guardian

- Comprehensive protocol

- The process of dialogue should be multi-stage (avoid packing too much information into one meeting with the patient)

- Consent remains valid for an indefinite duration unless it is withdrawn by the patient. Additionally, one must respect the right of the patient to refuse treatment and document the same at all times 
- Consent should be voluntary. Patients should be able to make decisions for themselves free from controlling interference and influences(12).

According to Ozar and Sokol (13), respecting patients' autonomy “yields satisfaction for that person [the patient] directly," while interfering with an individual's autonomy may be experienced as "a form of pain or suffering." 'This undoubtedly has contributed to a high level of trust between patients and the dental profession (14).

- Allow patient sufficient time to decide

- Document the fact that the patient has been given the opportunity to raise questions and doubts and that they have been answered.

- Adults are assumed to be competent unless demonstrated otherwise (7). Hence a clinician does not have to follow any special protocol or procedure to establish competency of a patient.

- Consent for additional procedures (sedation, bone grafting etc) should be obtained well in advance, not under duress with comprehensive information dispensed to the patient.

- Obtain consent for clinical photography and video recordings. The purpose and possible use of the video or photograph(s) must be clearly explained to the patient.

- Investigations should be a mirror image of the positive findings in the medical and dental questionnaires and the clinical examination of the patient.

- The Association of Dental Implantology in the United Kingdom (15) states “respecting patients' wishes particularly when patients may be involved in the training of other dentists". Explanations for the need for practical experience should be given to the patient; however it is not a legal requirement to tell the patient that the clinician is a student, although it would be good practice to do so. Clinician's owe a moral responsibility and not just an ethical one to the patients and for the sake of the former one should inform the patient about the status of training of the treating clinician.

\section{POSSIBLE CONSEQUENCES OF NOT OBTAINING CONSENT FOR TREATMENT}

Two areas of the law are relevant: trespass to person and negligence. If invasive treatment is provided without patient consent to the general nature of the procedure, then a practitioner may be sued for the tort of battery, and damages claimed for trespass to person - unless the failure to obtain consent is justified by necessity: for example, in an emergency. However, the role of the law of trespass in the area of 'informed consent' is limited. Consent to a procedure is not usually negated by being obtained without disclosure of associated risks and possible alternative treatments.

The most applicable sanction for failure to disclose this sort of information lies in the tort of negligence. It is expected that a practitioner's general duty to act reasonably includes a duty to provide adequate information, particularly in relation to risks or hazards. If something goes wrong then the practitioner may be exposed to liability for damages in negligence. A negligent act is usually found or alleged to have occurred in the procedure itself. However, a failure to provide information about the procedure and associated risks may also amount to negligence. For action in negligence on the latter score to succeed, two points must be established:

- That failure to disclose the information was unreasonable; and

- That this failure was a cause of harm to the patient.

The measure of reasonableness in relation to information-giving is akin to the standard of care required in relation to diagnosis and treatment, viz. that of an 'ordinary careful and competent practitioner of the class to which the practitioner belongs'.

To satisfy the second element (causation), the patient must establish both that he/ she would not have consented to the treatment had proper disclosure been made and that injury was suffered due to the treatment.
At present it is not easy for the plaintiff/ patient to establish any, let alone all of these things, especially causation. Actions in negligence are often unsuccessful. The mere fact of treatment without consent will not be regarded as compensatible injury. However, this may undergo change as the law in medical negligence evolves further, particularly in the areas of:

- Determining the weight that is to be accorded evidence derived from standard practice; and

- Assessing whether risk was material, that is, whether it would have influenced a reasonable person in the position of the patient in deciding whether to accept the procedure in question.

\section{DISCUSSION}

Is the very topic "Consent in dentistry" irrelevant? Is Consent the problem or a solution? Are we bringing upon ourselves an affliction of the west? Do we already have enough on our hands to worry about? The common denomination here is the word "WE". We, the professionals who safeguard the profession of dentistry; what about our patients? Are we not obligated to self-regulate!

Is all the above an overkill? To some, in our current climate it might be. Should we be ready for the negligence onslaught, which the western countries reel under? The answer would be a logical yes.

Dental law and dental ethics is an inevitable addition to the practise of dentistry. A sign of a mature society is one where the consumer or client is given the legal right to question and has an established framework in which to do so. This is as much protection for the client as it is for the dental professional. It's only prudent we ready ourselves. Dental law is here to stay.

\section{REFERENCES}

1. Merriam Webster's Dictionary

2. British Dental Association "Ethics in Dentistry" BDA 2000.

3. Glick M. Informed Consent: A Delicate Balance. J Am Dent Assoc 2006;137(8): 1060-62. 
4. D'Cruz L. Risk management in clinical practice: Part 2. Getting to 'yes' - the matter of consent. $\mathrm{Br}$ Dent $\mathrm{J}$ 2010;209:71-74.

5. Graskemper JP. A new perspective on dental malpractice: Practice enhancement through risk management. J Am Dent Assoc 2002;133(6):752-57.

6. Feigal RJ. Guiding and Managing the Child Dental Patient: A Fresh Look at Old Pedagogy. J Dent Educ 2001;65:136977.

7. King J. Consent: the patient's view - a summary of findings from a study of patient's perceptions of their consent to dental care. Br Dent J 2001;191:36-40.
8. Cronk EA, Cronk LS. Informed Consent in 2001: "Don't Leave the Office Without It". Published by the Royal College of Dental Surgeons of Ontario. June 2001.

9. Quarnstrom F. Risk Versus Cost. J Am Dent Assoc 2006;137:288b-290b.

10. Dudley N. Importance of risk communication and decision making in cardiovascular conditions in older patients: a discussion paper. Qual Health Care 2001;10(Suppl I):19-22.

11. Braddock $\mathrm{CH}$, Edwards $\mathrm{KA}$, Hasenburg $\mathrm{NM}$, et al. Informed decision making in an outpatient practice. J Am Med Assoc 1999;282:2313-20.

12. Beauchamp TL, Childress JF. Principles of Biomedical Ethics; 5th ed. New York City: Oxford University Press 2001:57-103.

13. Ozar DT, Sokol DJ. The relationship between patient and professional. In: Dental Ethics at Chairside: Professional Principles and Practical Applications. 2nd ed. Washington: Georgetown University Press 2002;49:53-54.

14. DiMatteo MR, McBride CA, Shugars DA, O'Neil EH. Public attitudes toward dentists: a US household survey. J Am Dent Assoc 1995;126(11):1563-70.

15. Association of Dental Implantology. The ADI Dental Implant Protocols. Produced by the Association of Dental Implantology, UK, 1998. 